\title{
Traumatic Avulsion Fracture of the Origin of the Brachioradialis Muscle: A Case Report and Review of the Literature
}

\author{
Dany k. Aouad Nabil Dib Chady Kharrat George El Rassi \\ Department of Orthopedic Surgery and Traumatology, Saint Georges University Medical \\ Center, Balamand University, Beirut, Lebanon
}

Keywords

Brachioradialis muscle · Avulsion · Trauma

\begin{abstract}
Avulsion fracture of the brachioradialis muscle origin at the proximal two-thirds of the lateral supraepicondylar ridge of the humerus is an extremely rare fracture with only 4 reported cases in the literature so far. In this article, we describe the case of a 50-year-old male patient who had sustained a closed fracture of the latter after falling from a height of $1.5 \mathrm{~m}$ on a flexed elbow and pronated hand. The patient was managed with immobilization of the elbow and wrist with a posterior splint in neutral position for 3 weeks, followed by wrist and elbow therapeutic mobilization sessions for 3 months. At the final follow-up, the patient had painless active and passive full range of motion of the elbow along with good flexion strength. The unique mechanism by which this avulsion fracture occurred is explained on the basis of the mode of injury, position of the limb, and structure and function of the brachioradialis muscle.
\end{abstract}

\section{Introduction}

The avulsion fracture of the origin of the brachioradialis muscle remains a rare injury. The infrequency of the presentation of this type of injury along with its distinct mechanism of injury makes it difficult to diagnose and further complicates management options. Only few 
cases have been reported in the English literature $[1,2]$. In this report, a case of a brachioradialis muscle origin avulsion fracture is presented with comparison of its mechanism of injury to other reported cases while discussing the different management options along with a brief literature review.

\section{Case Presentation}

A 50-year-old previously healthy male right-handed firefighter presented to the emergency department with severe right elbow pain after trying to stop a forward fall on a flexed elbow and pronated hand from a height of $1.5 \mathrm{~m}$. The patient attributes falling in pronation with a flexed elbow to water hose handling, having no time to adjust the arm to land in a correct position. The patient presented holding his arm in elbow flexion along with forearm supination. There was no obvious deformity, redness, or ecchymosis but only minimal swelling at the elbow region. On physical examination, the patient's right elbow flexion strength was decreased with inability to flex the elbow for $>15^{\circ}$ from the baseline position, with both active and passive ranges of motion limited by pain, but active extension could be done with mild pain from the initial position. Minimal supination or pronation of the forearm induced pain. The pain was also aggravated by active flexion and extension at the wrist joint. The patient had normal neurovascular exam of the arm, forearm, and hand.

Plain radiographs of the right elbow were ordered and revealed a small 4-mm displaced fracture at the level of the proximal two-thirds of the lateral supracondylar ridge (Fig. 1). After viewing the radiographs, CT scan with 3D reconstruction (Fig. 2) was done and showed avulsion fracture of the brachioradialis origin facing the lateral aspect of the distal humeral shaft, at the level of the brachioradialis muscle origin. In addition to a nondisplaced fracture of the anterior aspect of the radial head, a radioclinical diagnosis of avulsion fracture of the right brachioradialis muscle from its origin at the proximal two-thirds of the lateral supracondylar ridge was made, and management was planned accordingly.

In view of the minimally displaced fracture, the patient was treated conservatively with immobilization of both the elbow and the wrist with a posterior splint in $90^{\circ}$ elbow flexion and midpronated wrist for 3 weeks. At follow-up (on splint removal $t=3$ weeks), the patient regained full range of motion but with noticeable pain on active extension of the elbow and with slight decrease in flexion strength compared to the contralateral side. The patient was started on an early mobilization physical therapy protocol, progressively advancing from passive range of motion to active resisted. Emphasis was made on flexion of the arm against resistance in the midpronated position with gradual increase in weight-bearing potential along with specialized stretching exercises to reach painless full range of motion. On 3-month follow-up, the patient had regained full painless passive and active range of motion of both the wrist and elbow, with equal bilateral upper limb strengths, with an excellent Mayo Elbow Performance Index of 95 over 100.

\section{Discussion}

By definition, an avulsion fracture occurs when a small chunk of bone attached to a tendon or ligament gets pulled away from the main part of the bone. In rare cases, if the bone fragment and main bone are too far apart to fuse naturally, surgery may be necessary to reunite them. Furthermore, the mechanism of injury is often associated with an external force, an eccentric contraction of the brachioradialis muscle, or a combination of both [3].

\section{Karger'}




\section{Case Reports in Orthopedic Research}

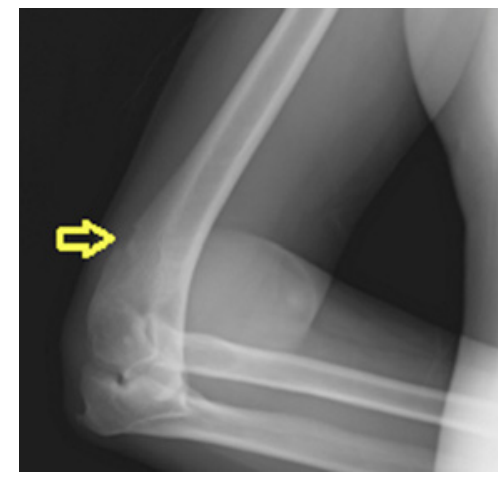

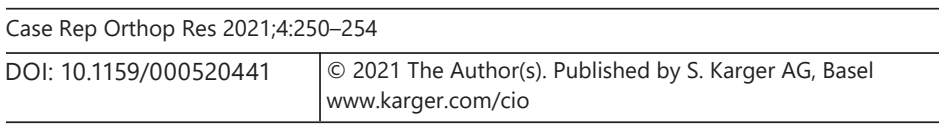

Aouad et al.: Traumatic Avulsion Fracture of the Origin of the Brachioradialis Muscle

Fig. 1. Plain radiographs showing multiple views of the right elbow with small 4-mm displaced fracture at the level of the lateral supracondylar ridge.

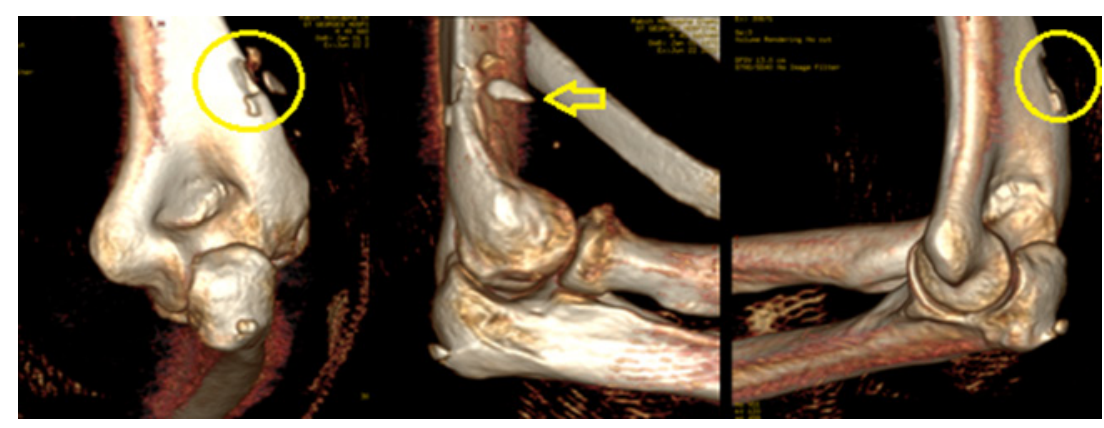

Fig. 2. CT scan 3D reconstruction views of the right elbow showing an avulsion fracture of the brachioradialis origin facing the lateral aspect of the distal humeral shaft, at the level of the brachioradialis muscle origin. In addition to a nondisplaced avulsion fracture of the anterior radial head, the coronoid process and the lateral humeral condyle.

The brachioradialis muscle originates at the anterolateral aspect of the distal humerus and is part of the dorsal mobile wad along with extensor carpi radialis longus and extensor carpi radialis brevis. Also, it inserts at the radial styloid, which along with its origin offers a mechanical benefit with elbow flexion especially when the forearm is at midpronation [4]. The attachment of this muscle is known to give it mechanical qualities making it a muscle rarely involved in avulsion fractures, and it is innervated by the radial nerve [5].

Given the passage of the radial nerve between the brachialis and the brachioradialis muscles at the antecubital fossa, the radial nerve and more specifically the superficial branches of it are at risk of injury even from the avulsion fracture itself. Assessment of patients with brachioradialis avulsion fracture should therefore include cautious evaluation of the radial nerve functions [5]. Radial nerve injuries remain a main complication in surgical procedures involving this part of the arm.

According to an electromyographic study published in 2017, the brachioradialis is one of the main flexors of the elbow, especially when the elbow flexion is done with the forearm in pronation, and it also plays an important role in the dynamic stabilization of the elbow [6]. This could explain the slight decrease in elbow flexion strength the patient had when the arm was pronated after this injury. Furthermore, in another electromyographic study, it was shown that brachioradialis had more intense activity during elbow flexion and less activity in elbow extension [7], which can also explain how the patient was able to actively extend his elbow with no deterioration in strength after the injury. In this case, the mechanism of injury 
is due to direct trauma to the elbow in flexion which is different from those reported in the literature. This may be explained by the increased activity of the muscle during concentric flexion of the elbow which in addition to the trauma led to the avulsion fracture.

In contrast, most commonly, the location of avulsion fractures on the humerus occurs with fractures of lateral or medial epicondyles or fractures of the greater or lesser tuberosities. Therefore, in total, only 4 cases of avulsion fractures of the brachioradialis muscle have ever been described in the literature since the first one in 2001.

In 3 of the previously reported cases, the mechanism of injury was almost the same with the patient trying to prevent a backward fall, on an outstretched arm with the elbow and shoulder extended and the forearm pronated. The fall on the arm in this position lead to the eccentric contraction of the brachioradialis muscle leading to the avulsion injury to occur at its origin $[3,4]$.

In contrast to the other reports, in the above-described case, the patient tried to prevent a forward fall with a flexed elbow and pronated hand which similar to the mechanism of injury discussed in the previous articles led to the eccentric contraction of the brachioradialis muscle leading to the avulsion injury, thus adding to the literature that such avulsion fractures can occur with forward falls as well, and that it is the action of the overwhelming contraction of the brachioradialis muscle whether concentric or eccentric that leads to this type of injury.

The patient presented with a minimally displaced closed avulsion fracture at the level of the proximal two-thirds of the lateral supracondylar ridge in the right humerus with no sensory or motor deficit. The patient was not a candidate for surgery since he had a minimally displaced avulsion fracture. Conservative management was opted, with immobilization of the elbow and wrist joints, followed by early mobilization to prevent stiffness. Surgical options comprise a high risk of injury to the radial nerve in view of its close proximity to the avulsion fracture fragment apart from the implant-related complications and the need for second surgery to remove the implants [3].

\section{Conclusion}

Cases of avulsion fractures of the brachioradialis muscle origin are scarcely described in the literature, and thus no clear algorithm for the appropriate treatment is available. However, the above case showed a good outcome following conservative treatment.

\section{Statement of Ethics}

Approval and consent of the ethics committee institutional review board (IRB) has been received for the publication of this article (no reference number available). Written informed consent was obtained from the patient for publication of this case report and any accompanying images.

\section{Conflict of Interest Statement}

The authors declare that they have no competing interests.

\section{Funding Sources}

No funds were received in support of this study.

\section{Karger'}


Case Reports

in Orthopedic

Research
Case Rep Orthop Res 2021;4:250-254

\begin{tabular}{l|l}
\hline DOI: $10.1159 / 000520441$ & (c) 2021 The Author(s). Published by S. Karger AG, Basel
\end{tabular} www.karger.com/cio

Aouad et al.: Traumatic Avulsion Fracture of the Origin of the Brachioradialis Muscle

\section{Author Contributions}

D.A. contributed to writing and editing the article. N.D. contributed to writing and editing the article. C.K. contributed to writing and editing the article, with editing of the images and radiographs. G.E.R. is the surgeon who supervised writing and finalizing the article.

\section{Data Availability Statement}

The datasets used and/or analyzed during the current study are available from the corresponding author on reasonable request.

\section{References}

1 Guettler JH, Mayo DB. Avulsion fracture of the origin of the brachioradialis muscle. Am J Orthop. 2001;30(9): 693-4.

2 Marchant MH, Gambardella RA, Podesta L. Superficial radial nerve injury after avulsion fracture of the brachioradialis muscle origin in a professional lacrosse player: a case report. J Shoulder Elbow Surg. 2009;18(6): e9-12.

3 Behera G, Balaji G, Menon J, Sharma D, Komuravalli VK. Avulsion fracture of brachioradialis muscle origin: an exceedingly rare entity: a case report. Malays Orthop J. 2016;10(2):50-2.

4 An KN, Morrey BF. Biomechanics of the elbow. In: Morrey BF, editor. The elbow and its disorders. Philaedlphia: WB Saunders; 1985. p. 43-61.

5 Marchant MH Jr, Gambardella RA, Podesta L. Superficial radial nerve injury after avulsion fracture of the brachioradialis muscle origin in a professional lacrosse player: a case report. J Shoulder Elbow Surg. 2009; 18(6): $99-12$.

6 Caufriez B, Dugailly PM, Brassinne E, Schuind F. The role of the muscle brachioradialis in elbow flexion: an Electromyographic Study. J Hand Surg Asian Pac Vol. 2018;23(1):102-10.

7 Nakazawa K, Kawakami Y, Fukunaga T, Yano H, Miyashita M. Differences in activation patterns in elbow flexor muscles during isometric, concentric and eccentric contractions. Eur J Appl Physiol Occup Physiol. 1993; 66(3):214-20. 